\title{
Comet: A naval Combat Management System model for combat analysis
}

\author{
$\underline{\text { S.G. Blaess }}^{\text {a }}$, M.J. Christie ${ }^{\text {a }}$ and J.L. Mitchell ${ }^{a}$ \\ ${ }^{a}$ Weapon and Combat Systems Division, Defence Science and Technology Group, Edinburgh, South \\ Australia \\ Email: simon.blaess@dst.defence.gov.au
}

\begin{abstract}
A Combat Management System (CMS) is the computers and software of a naval platform which integrates the sensors, weapons, displays, data and other equipment, enabling the naval platform to operate efficiently and effectively to achieve mission success. In a hostile environment where threats may be fast moving, hard to detect, large in number, or all of the above, a crewed naval surface vessel requires a CMS that not only acts at the behest of the crew, but can also act autonomously when required. The capacity of the CMS to utilise data, respond autonomously, provide information to the crew and respond to crew commands significantly impacts the ability of the platform to achieve its mission. Modelling and simulation of not just the ship's sensors and weapons, but also the CMS, is therefore critical if we aim to thoroughly evaluate the capability of the platform in combat scenarios. Such studies can reveal the strengths and weaknesses of a platform, capability gaps and areas of opportunity, providing an evidence base to improve tactics and strategy, force structure and acquisition decisions.
\end{abstract}

As of 2021, the Royal Australian Navy surface fleet includes three Hobart Class guided missile destroyers, each employing the Lockheed Martin developed Aegis CMS. The Royal Australian Navy also comprises eight Anzac Class frigates employing the Saab developed 9LV CMS. Later this decade the Anzac Class frigates will begin to be replaced by nine Hunter Class frigates with an Aegis CMS and 9LV tactical interface. Understanding the capability and limitations of these ships requires an understanding of how their respective CMS installations will perform. Based on references of the Aegis CMS, we have constructed, and continue to develop, a highly configurable CMS model called Comet, which is capable of interfacing with other models in constructive simulation environments to enable naval combat analysis studies. The architecture and functionality of Comet are presented in this paper.

Comet has been utilised in combat analysis studies to evaluate the performance of surface naval platforms against a variety of missile threats. The Find, Fix, Track, Target, Engage, Assess (F2T2EA) kill chain summarises the sequence of processes which occur in the detection, engagement and intercept of a threat. By evaluating the F2T2EA kill chain, the performance capabilities and limitations of a platform are identified and opportunities for improvement in the engagement kill chain are discovered. Alternative tactics, strategy, equipment configurations and/or new procurements can be modelled and analysed to discover if they improve capability.

Comet can be executed in two different modes: the CMS Emulation mode or the System Level Analytical Baseline (SLAB) mode. The SLAB mode is a cut-down version of the Comet Fire Control System developed to provide an efficient and comprehensive evaluation of threat engageability and interceptability at each point in time, whereas the CMS Emulation mode represents how a real CMS will engage the threat, wait for the intercept attempt to complete and then reengage if necessary. The analytical use cases of Comet are presented here in the context of analysing the air and missile defence capability of naval surface vessels.

Keywords: Combat management system, integrated modelling, modelling and simulation, F2T2EA, missile defence 


\section{INTRODUCTION}

A strong modelling and simulation (M\&S) Defence capability is important in providing Defence with the evidence base to make informed decisions. Training and readiness, force development and force structure can all be improved via the appropriate use of M\&S. In the context of Australia's naval capability, modelling and simulation of naval components and/or assets can enhance the operation of Australia's naval resources, improve acquisition decisions and reveal capability gaps and risks.

The Royal Australian Navy's surface capability includes three Hobart Class guided missile destroyers (DDGs) and eight Anzac Class frigates. Towards the end of this decade the Anzac Class frigates will begin to be replaced by nine Hunter Class frigates (Department of Defence 2016). Each ship has a Combat Management System (CMS) which integrates the ship's array of sensors, weapons, displays and other equipment and data such that the platform is able to be managed and operated via automated actions and operator interaction. The Hobart Class DDGs operate the Aegis CMS whilst the Anzac Class frigates operate the Saab 9LV CMS. The Hunter Class frigates will operate the Aegis CMS with a 9LV tactical interface used predominantly to integrate bespoke Australian capability. The capability of the platform in hostile environments is impacted by the ability of the CMS to integrate the platform's equipment, use data to make decisions, provide information to the crew and effectively respond to operator control or act autonomously. Thorough analysis of naval platform capability therefore requires the CMS to be appropriately represented in M\&S tools, in addition to the ship's sensors and effectors. CMS models can be used to improve tactics and strategy, identify better equipment configurations and help inform future naval asset acquisitions.

We have developed a highly configurable naval CMS model called Comet. This model can interface with other models in constructive simulations to facilitate combat analysis studies (van Bodegom et al. 2019; Power et al. 2021). In this paper we describe the CMS model architecture and its functionality. The uses of Comet in the simulation and analysis of naval surface vessels in an air and missile defence context are also presented.

\section{COMET ARCHITECTURE}

Comet is a $\mathrm{C}++$ model built using the MarsGen toolkit (Fletcher et al. 2009), a Defence Science and Technology Group (DSTG) model development framework which generates base $\mathrm{C}++$ model code so that effort can be focused on developing the functionality of the models. Comet is wrapped within SimFramework 5 and OneWorld (Armstrong et al. 2021), a DSTG ecosystem for integrating C++ models into constructive simulation environments such as Missile Engagement and Coverage Analysis (MECA), a DSTG software tool for performing complex engagement level combat analysis.

Comet's architecture is based on the available reference material of the CMS installed on the Royal Australian Navy's air warfare destroyers and frigates. Comet is divided into major functional components called domains that each encompass a basic functional responsibility of the CMS, for example the management of weapons or the management of sensors. Each domain consists of a set of models that execute the required tasks of the domain. This modular approach allows components of Comet to be updated and integrated more easily, whilst also enabling the configurability of each component. A real CMS would contain additional domains, for example a Display domain which provides interfaces to the operators, but these are of limited relevance in a constructive simulation.

\section{OneWorld}

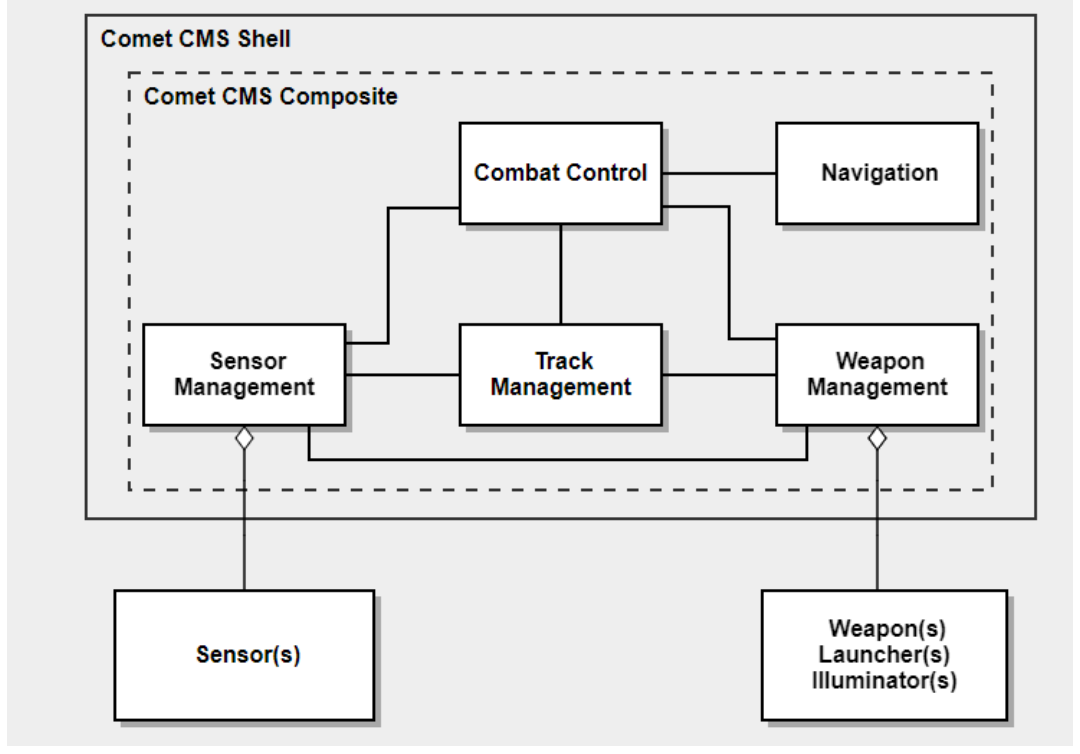

Figure 1. A simplification of the OneWorld ecosystem and the domains of Comet. 
A high-level overview of Comet and the OneWorld ecosystem is shown in Figure 1. Within the OneWorld ecosystem, but outside Comet, are the resources that Comet is responsible for managing and tasking, such as the sensors, weapons, launchers and illuminators. The Comet Shell (or wrapper) translates outgoing signals into a OneWorld compatible format so that these resource models residing in OneWorld can be instructed by Comet. Similarly, the Comet Shell translates incoming OneWorld signals, allowing Comet to receive and interpret data such as the status of resources.

\section{COMET DOMAINS}

The domains of Comet and their current functionality are summarised below.

\subsection{Combat Control}

The Combat Control domain handles tactical decision making and determines courses of action by processing information requested and received from the other domains.

Comet's Combat Control domain evaluates and prioritises threats, requests engagement options from the Weapon Management domain and then processes the received engagement options. This culminates in engagement orders that are sent to the Weapon Management domain for action. Comet's Combat Control domain also receives engagement status updates from the Weapon Management domain in case a threat needs to be re-engaged. Further development of the Combat Control domain will include configurable tactical doctrine to enable autonomous responses to engagement situations.

\subsection{Sensor Management}

A complete Sensor Management domain would monitor and report on sensor functionality, manage sensor tasking, control the sensors and handle sensor track data.

Comet's Sensor Management domain is currently of limited functionality. It registers basic sensor capability information that is then passed to the Combat Control domain to facilitate decision making. It also passes on source track data from the sensor resources to the Track Management domain. Further work will expand the functionality of this domain.

\subsection{Track Management}

The Track Management domain receives track data originating from sensor resources and networks, and then processes this data to form a common tactical picture comprised of system tracks. The Track Management domain provides other domains access to system tracks via a track server. Comet's Track Management domain achieves the above functionality, but does not currently implement track filtering, correlation or fusion. Comet instead creates or updates system tracks directly from the source tracks of sensors. The true identity of the source track is checked to ensure duplicate system tracks of an entity are not created. Track filtering, correlation or fusion can however be performed within the sensor resource.

\subsection{Weapon Management}

The Weapon Management domain handles the missile, launcher and illuminator resources of the combat system to support engagements. This domain collects resource availability and capability information, evaluates engagement requests, schedules and actions engagements, and evaluates and reports on the status of engagements. Comet's Weapon Management domain is well developed in regards to missiles and launchers. The illuminator functionality of Comet is currently under development to include illuminator scheduling constraints.

The Weapon Management domain primarily consists of resource controller models connected to an Air and Missile Defence (AMD) Coordinator model and Engagement Scheduler model. Figure 2 displays a simplification of the signals within Combat Control and Weapon Management that culminate in an engagement. The engagement process can be conceptualised as two phases; a request phase and an order phase. While a threat is tracked, the Engagement Manager sends an engagement request to the AMD Coordinator. Upon receiving a request the coordinator asks the Resource Controllers to evaluate their capability to engage. In the case of a launcher controller, this simply involves checking that there are enough missiles in the inventory. A missile controller however will need to perform an engageability calculation, which evaluates the capability of its type of missile versus the threat and its estimated future trajectory, to reach a conclusion on 


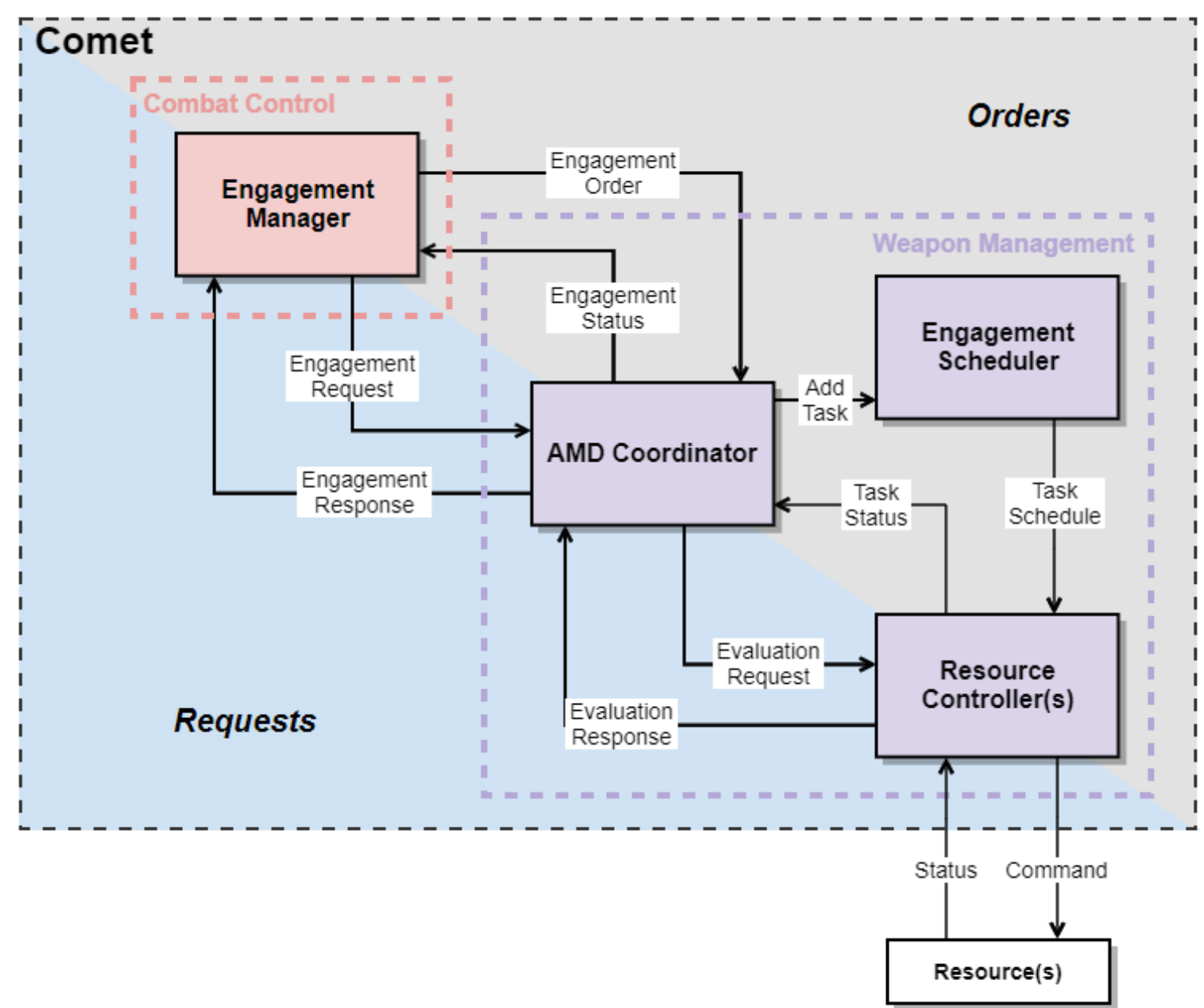

Figure 2. A simplified overview of the signals within Comet's Combat Control domain and Weapon Management domain that culminate in an engagement.

whether the threat can be intercepted and, if so, the time and location of intercept. The coordinator collects the evaluation responses from the controllers and decides on the best option, providing an engagement response to the Engagement Manager. If the response is sufficient for engagement to proceed, an engagement order is sent to the AMD Coordinator. The coordinator uses the best option to create a task which is sent to the Engagement Scheduler. Using knowledge of each resource's capability, it is the responsibility of the Engagement Scheduler to continuously compile a task schedule which does not result in resource conflicts. The updated task schedule is continually sent to the resource controllers, which will act on these tasks when required. For example, a launch task sent to the launcher controller will result in a launch command sent to the launcher resource at the appropriate time. The resources and resource controllers are able to provide status updates, which flow back up the chain to inform the AMD Coordinator, Engagement Manager and any other relevant models of the progress of an engagement.

An important component of the Weapon Management domain are the target prediction algorithms used to predict where a threat will be at a future time. These are used by the missile controllers when evaluating threat engageability and potentially when a missile is in flight. The selected target prediction algorithm will significantly influence whether a threat is deemed engageable and whether the time of intercept and predicted intercept point (PIP) are accurate. If a missile is launched, the missile controller is able to provide target state and PIP updates to the weapon as the engagement progresses, allowing the weapon to consider the latest information and manoeuvre towards the threat. Alternatively, the weapon can use its own guidance logic postlaunch, for example adopting a proportional navigation solution to its own PIP. 


\section{System Level Analytical Baseline}

The System Level Analytical Baseline (SLAB) mode is essentially a simplification of the Weapon Management domain that enables a more comprehensive assessment of the complete engagement timeline, whilst also aggregating data in a simple csv format. Instead of the Weapon Management domain going through the full process of evaluation requests, evaluation responses, receiving engagement orders and forming an engagement schedule, the SLAB mode of Comet will evaluate threat engageability, and if the threat is engageable a launch command is immediately sent to a launcher. This results in missile firings at every time step the threat is deemed engageable, allowing the engageability and interceptability of the threat to be evaluated over time. Additionally, the SLAB mode of Comet will output a csv file that contains the time, target position and velocity, predicted intercept point (if one exists), and other data of interest such as whether the threat was above a specified speed threshold or if the threat would be below the horizon at the PIP. This csv output provides convenient access to engageability timeline data.

\subsection{Navigation}

Comet currently has a skeleton Navigation domain that simply outputs the ship's platform state. To assist firing and illumination arcs, this domain will eventually include manoeuvre or steering commands that are sent to a ship motion model.

\section{COMET CONFIGURATION, SIMULATION AND ANALYSIS}

\subsection{Configuration}

Comet is designed to be highly configurable via configuration files that define the models to be executed and their behaviour.

\section{Model behaviour}

Each Comet model is parameterised, allowing the configuration files to define aspects of how the model will execute. The model execution rate, time delays for particular processes, target prediction algorithms used, threat prioritisation approach, engageability checks or how kill-evaluation is triggered are examples of model behaviour that can be configured.

\section{Number of entities}

There are no fixed requirements on the number of sensor, launcher, missile or illuminator resources that must be linked to Comet. If a resource is excluded, its related controller can be excluded from Comet. Similarly, if multiple instantiations of the same resource type exist, multiple controllers can be instantiated and each can have their own unique behaviour. For example, if two classes of missile resources are attached to the platform, two separate missile controller models can be instantiated, which use different target prediction algorithms.

\subsection{Simulation and Analysis}

The modular and configurable nature of Comet facilitates simulations that can investigate a range of platform capabilities. Comet has thus far been used for engagement level combat analysis, where the ability of the platform's sensors, weapons and CMS to intercept an incoming threat are investigated. The performance of Comet has been validated using trials data.

The Find, Fix, Track, Target, Engage and Assess (F2T2EA) kill chain summarises the basic steps towards engaging a threat (Department of Defence 2009). Figure 3 visualises the kill chain, with the bold text indicating the sub-tasks of the kill chain steps that are currently modelled by Comet. The analysis of Comet simulation data can be used to evaluate the success of the CMS at engaging threats and identify where in the kill chain capability may be lacking, thus revealing potential areas for improvement. For example, if Comet was unable to receive any threat tracks, this reveals that local and/or external sensors were unable to search or detect the threat. Altering the configuration of the sensor or employing a sensor with greater power may address this issue. If the platform was able to find, fix and track the threat, but the CMS did not deem the threat engageable or only within a narrow time window, this may indicate the configured target prediction algorithm and/or 

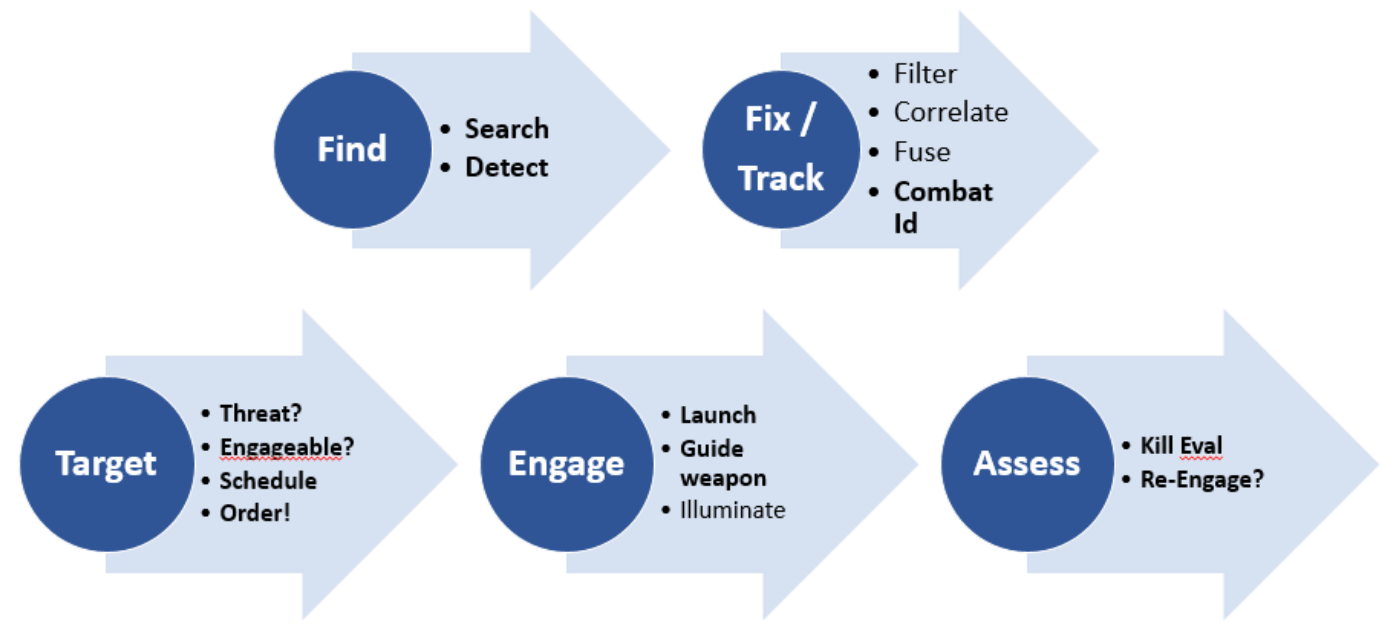

Figure 3. Summary of the F2T2EA kill chain. In bold text are the aspects that are currently modelled in Comet.

ownship weapon assumptions are inadequate, delays in the processing of tasks are impacting the ability of the platform to engage, and/or the platform's weapons are simply overmatched against the threat.

An example application of the

SLAB mode of Comet is displayed in Figure 4, which shows a hypothetical missile threat trajectory and when a fire control solution (FCS) is able to be found. A FCS means the platform has determined a weapon launch will intercept the threat when fired upon at that location. If a FCS exists then a corresponding PIP for that launch is shown (note the 16 PIPs shown by green crosses correspond to the 16 FCSs shown by green dots). The region where this weapon may be able to intercept a threat (i.e. the region where PIPs could exist) is the weapon engagement zone (WEZ). In Figure 4 we see a window of opportunity exists

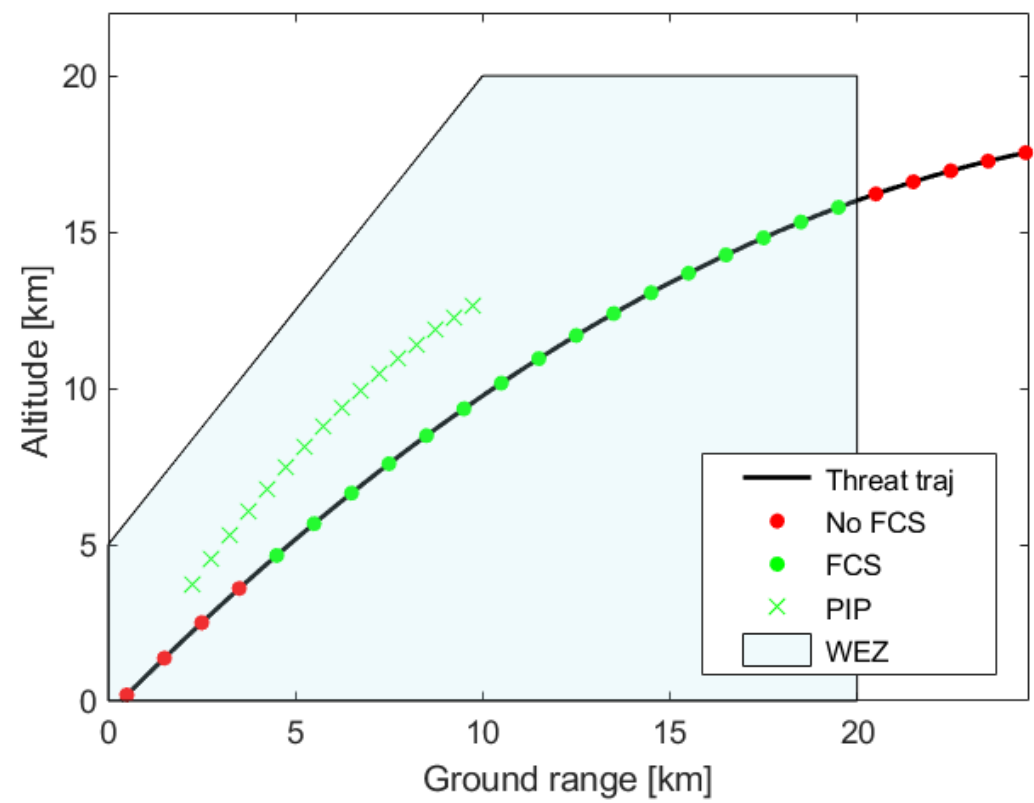

Figure 4. An example plot derived from SLAB data revealing the engageability of a threat. where the platform expects that a launch will intercept the threat. Depending on the configuration of Comet, for example the target prediction algorithm chosen, there may be drastic differences in the ranges at which the threat is engageable and the location of the resultant PIPs. In Figure 4 the target prediction algorithm is able to reasonably approximate the true threat trajectory, resulting in PIPs at launch time that are sufficiently close to the threat's future trajectory. Upon launch the weapon model can be provided with in-flight PIP updates that may trend closer to the threat's true trajectory as the engagement progresses. Alternatively the weapon may employ its own guidance logic. The performance of different target prediction and guidance logic will depend on the speed and manoeuvrability of the threat. 


\section{CONCLUSION AND FUTURE WORK}

This paper presents a naval surface vessel CMS model that can be interfaced with other models in constructive simulations to perform combat analysis. The highly configurable design of this model allows a range of capabilities and scenarios to be analysed. A cut-down version of the CMS model, called the SLAB mode, can be utilised to obtain more comprehensive engageability timeline information.

The functionality and capability of Comet's domains is under continual development. Furthermore, the simulation framework underneath Comet is being re-evaluated. Comet functionality is currently represented within a hierarchical model breakdown aligning with the domains described earlier. It defines point-to-point input and output pathways that determine where a model sends a signal. We are aiming to transition to a publish-subscribe messaging paradigm, where a model can publish any type of signal and a model can opt to subscribe to any signals it deems relevant for its function. This will better align Comet with how a real CMS operates and enable Comet to be integrated into virtual simulations for human-in-the-loop studies. Aligned with this transition is a desire to increase the environment portability of Comet so that individual Comet models can be easily implemented within another model, framework or simulation, instead of implementing the entire Comet CMS model configured to execute only the models of interest.

\section{REFERENCES}

Armstrong, R.D., Christie, M.J., Mellen, P.R., 2021. OneWorld: Evolving an architecture for complex system representation in engagement simulation. 24th International Congress on Modelling and Simulation, Modelling and Simulation Society of Australia and New Zealand.

Department of Defence, 2009. Operations Series ADDP 3.14 Target. Australian Defence Doctrine Publications, Chapter 5.

Department of Defence, 2016. 2016 Defence White Paper.

Fletcher, D., Hodson, M., Luckman, N., 2009. Advances in simulation architecture-independent model development. SimTecT 2009 Simulation Conference: Simulation - Concepts, Capability and Technology, https://stpubs.dsto.defence.gov.au/handle/dst/59530.

Power, W., Wylie, M., Mellen, P., van Bodegom, P., 2021. A Hybrid between Model-Based Systems Engineering and Agile Methodologies for Simulation of Complex Weapon Systems of Systems. IEEE Aerospace Conference.

van Bodegom, P., Blaess, S.G., 2019. Investigating the use of uninhabited aerial systems (UAS) to support anti-ship missile defence through simulations. 23rd International Congress on Modelling and Simulation, Modelling and Simulation Society of Australia and New Zealand. 